\title{
Effect of transverse strains and angular distortions on the nanoscale elastic behavior of platelet nanocomposites
}

\author{
G. Fernández Zapico ${ }^{a}$, J.M. Munoz-Guijosa ${ }^{a}, *$, Benito del Río ${ }^{a}$, Hiroki Akasaka ${ }^{b}$ \\ 'Mechanical Engineering Department, Universidad Politécnica de Madrid, Spain \\ ${ }^{\mathrm{b}}$ Mechanical Engineering Department, Tokyo Institute of Technology, Japan
}

\section{A R T I C L E IN F O}

\section{Keywords:}

Computational modelling

Polymer-matrix composites (PMCs)

Debonding

\begin{abstract}
A B S T R A C T
In order to correctly predict the macroscale elastic behavior of nanocomposite macroscale structures, an accurate nanoscale model must be available for subsequent homogenization. In this work, we demonstrate that the accuracy of that nanoscale model greatly depends on the consideration of transverse strains and angular distortions, which are not frequently taken into account, but have a significant influence on the cohesive mechanisms at the nanofiller-matrix interface. We use a nanoscale cohesive model to qualitatively and quantitatively analyze the effect of transverse shear and angular distortion on the interfacial stress transfer mechanisms. While the effect of the transverse strain is less significant, results show that angular distortion greatly affects the interfacial damage pattern. It appears to shift the interfacial shear stress distribution to one of the interface ends, which consequently also modifies the interfacial longitudinal stress distribution and its mean value, resulting in reduced nanocomposite stiffnesses. The effect should be taken into account as shear and transverse strains may be present at the macroscale if, for instance, nanofiller misalignment or stress concentrators exist. We also provide design maps representing damage onset for different 2D multiaxial strain states in graphene-epoxy nanocomposites, so that the strain state limit can be inferred for the given nanocomposite properties. A substantial reduction in the allowable strains can be observed.
\end{abstract}

\section{Introduction}

Due to the impressive properties of carbon and other 1D and 2D nanoparticles, considerable enhancements are to be expected when small amounts of these materials are added to different matrices [1 4]. Interesting improvements in mechanical properties have been achieved for nanofiller weight fractions as small as $0.5 \%$ [5 8]. In order to allow for the design of complex structures and geometries based on nano composites, constitutive equations must be available that take into account these effects, along with those appearing at the meso or mac roscale as filler misalignment or suboptimal filler dispersion, so that they can be introduced in the analysis codes used by mechanical de signers.

Different material models have been developed that predict nano composite stiffness, with some based on molecular dynamics [9 11], and others using inclusion methods such as Eshelby's tensor [12] or multiscale modelling $[13,14]$ At the nanoscale level, multiple papers have pointed to debonding as the main failure mechanism $[15,16]$. Due to its fundamental influence on nanocomposite mechanical properties [17 20], constitutive models must consider the interfacial stress transfer mechanisms at the filler/matrix interface. Firstly, given that the nanofiller matrix interface has a finite stiffness, the nanofiller strain will be smaller than that theoretically obtainable with a perfectly rigid interface. Secondly, since interfacial strength is finite, excessive inter facial separation may lead to interfacial damage. Both of these issues can lead to a decrease in interfacial stress transfer capacity and the nanocomposite properties will diverge from those obtainable if the in terface had infinite stiffness and strength. This can result in mechanical properties that are frequently below those that are theoretically achievable $[5,21]$.

Cylindrical carbon nanofiller epoxy interfaces are studied in Ramdoum et al. [17] by means of a cohesive model to model the in terfacial stiffness and damage. The stress transfer mechanisms at work in the elastic, debondable filler matrix are also well explained in pla telets composites by Heidarhaei et al. [18], Gong and Guo [19,20] or Ang et al. [22]. Those models demonstrate that when a strain is applied to the matrix parallel to the nanoparticle longitudinal axis, the nano particle is also strained by the shear created at the nanoparticle matrix interfaces due to the difference in stiffness. Rahman et al. [23] studied the interfacial properties and the effect of nanofiller dispersion using

\footnotetext{
* Corresponding author.

E-mail address: JMGuijosa@etsii.upm.es (J.M. Munoz-Guijosa).
} 

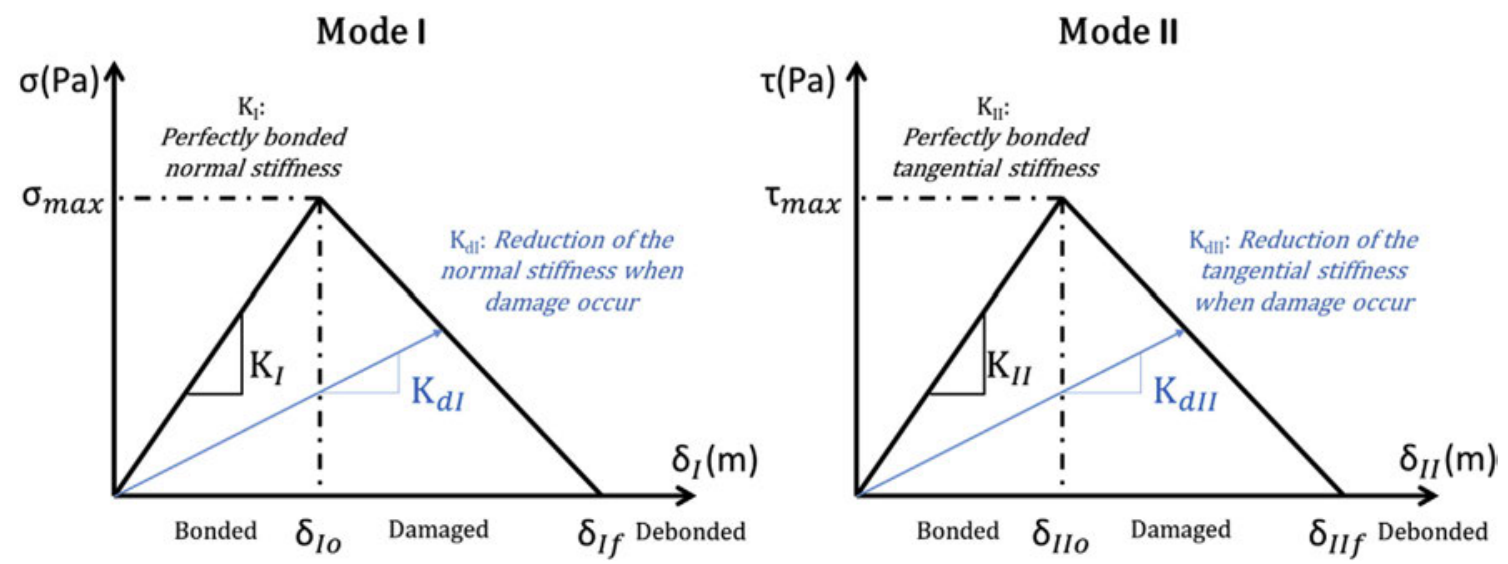

Fig. 1. Cohesive Zone Models (CZM) associated to frature mode I (left) and fracture mode II (right).

molecular dynamics. Similarly, Chen and Yan [24] proposed an ana lytical fiber pull out model based on a shear lag model with cohesive interfaces.

The above analytical considerations have all been experimentally validated. Jiang et al. [25] and Lee et al. [26], measured the strain field in a single graphene layer on the surface of an epoxy matrix; while the matrix was gradually strained they observed certain strain dependent Raman band shifts. Cui et al. [27] also used Raman spectroscopy to measure strain in carbon nanotubes to infer the interfacial character istics. Experimental results have a close correlation with the theoretical predictions for interfacial shear strengths of about $0.5 \mathrm{MPa}$ and fracture energies of about $0.08 \mathrm{~N} / \mathrm{m}$.

However, all the aforementioned models and experiments are based on uniaxial strain states. This assumption, which can be experimentally reproduced and modelled at the nanoscale, has led to many useful and interesting results that have provided a better understanding of na noscale mechanisms. However, at the macroscale, nanoparticles will be subjected to a multiaxial strain state if, for instance, they are not per fectly aligned with any of the principal strain fields or are close to stress concentrators. As shear and transverse strains may not be negligible in those situations, correct constitutive models should consider them at the nanoscale; they may have an influence on the interfacial mechan isms and consequently on the nanocomposite properties.

Few cohesive models have been found that are able to analyze the interfacial mechanisms for multiaxial strain states [28,29]. use an in verse analysis for their study and do not, therefore, focus on the in terfacial stress state. Belabed et al. [30] introduce shear and normal deformation in functionally graded materials. Experimentally, the problem is even more complex due to the difficulty of isolating and applying multiaxial strains to a single particle which, additionally, must not be located on a matrix free surface. This requirement also poses an important problem for the Raman measurement. At the macroscale level, Lee et al. [31] have studied biaxial behavior in a polymer steel interface by means of a cohesive model. Data was obtained using a three point bending test. However, the results cannot be extrapolated at the nanoscale due to the reinforcement stiffness and aspect ratio dif ference.

In this work, we present a nanoscale cohesive model for platelet nanocomposites that considers a $2 \mathrm{D}$ multiaxial strain state, so that the influences of angular distortions and transverse strains can be studied. The goal is to provide the scientific community with design tools that can be useful to determine the needed nanocomposite properties for a given strain state.

In section 2 we describe the model developed. In section 3 , we present a parametric study and qualitatively and quantitatively explain the effect of shear and transversal strains taking a graphene epoxy nanocomposite as an example. In section 4 , we provide some maps that can be useful to determine the feasibility of certain nanocomposite properties for a given strain state. Finally, the conclusions are presented in section 5 .

\section{Model description}

Traditional cohesive models were used as the basis (1) [19,32,33]:

$\sigma_{x}=f\left(\varepsilon_{C}, l_{F}, t_{F}, v_{F}, E_{F}, G_{F}, E_{M}, G_{M}, k_{I I}, \tau_{a}, G\right)$

where $\sigma_{x}$ is the longitudinal stress along the nanofiller length for a given uniaxial applied strain $\varepsilon_{C}$ at the nanocomposite, $l_{\mathrm{F}}$ the nanofiller length, $t_{F}$ the nanofiller thickness, $v_{F}$ the nanofiller volumetric fraction, $E_{F}, E_{M}$, $G_{F}$ and $G_{M}$ the nanofiller, matrix, Young's modulus and shear modulus, respectively; $k_{I I}$ the in plane interfacial stiffness, $\tau_{a}$ the interfacial shear strength and $G$ the interfacial fracture energy. We have introduced an applied angular distortion $\gamma_{x y}$ and an applied transverse strain $\varepsilon_{y}$. These additional strains will produce additional shear stress at the interface, as well as a transverse stress $\sigma_{y}$, with the corresponding Poisson effect on the longitudinal axis. For the calculation of the transverse stress state, additional interfacial parameters must be considered: transverse interfacial stiffness $k_{I}$ and transverse strength $\sigma_{a}$. The resulting model is described in eq. (2).

$\left[\sigma_{x}, \sigma_{y}, \tau_{x y}\right]=f\left(\varepsilon_{x}, \varepsilon_{y}, \gamma_{x y}, l_{F}, t_{F}, v_{F}, E_{F}, G_{F}, E_{M}, G_{M}, k_{I I}, \tau_{a}, k_{I}, \sigma_{a}, G\right)$

For both in plane and transversal directions, we use a bilinear in terfacial stress interfacial separation curve. Fig. 1 shows the bilinear constitutive laws for each fracture mode. $K$ is the interface stiffness, $\delta$ is the actual matrix nanofiller separation, $\delta_{0}$ is the separation at the onset of interfacial damage, $\delta_{f}$ the separation needed to fully debond the filler matrix interface, $\tau$ and $\sigma$ the stresses appearing at the interface and $G_{c}$ the area under the lines, which corresponds to the interfacial fracture energy. For any of the fracture modes taken individually, eqs. (3) (6) apply.

$\delta_{I 0}=\frac{\sigma_{\max }}{K_{I}} \quad \delta_{I I 0}=\frac{\tau_{\max }}{K_{I I}}$

$\delta_{I f}=\frac{2^{*} G_{i}}{\tau_{\max }} \delta_{I I f}=\frac{2^{*} G_{i}}{\sigma_{\max }}$

Damage $=\left(\frac{\delta_{i}-\delta_{i 0}}{\delta_{i}}\right)\left(\frac{\delta_{i f}}{\delta_{i f}-\delta_{i 0}}\right)$

$K_{i N E W}=K_{i}(1-$ Damage $)$

As both in plane and transverse interfacial separations may si multaneously exist, we use a fracture mode I and fracture mode II mixed mode debonding method as suggested by Ref. [32,33]. This implies the use of eq. (9) for defining a unique, equivalent interfacial damage from two interfacial displacements [34], being $\delta_{m i}$ the mixed 


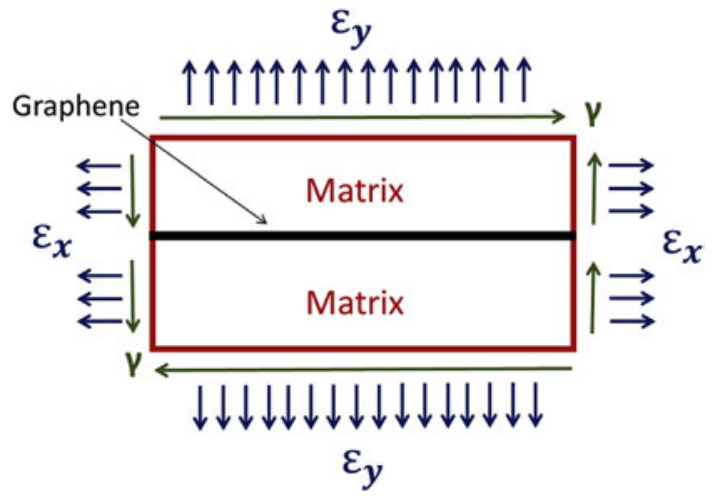

Fig. 2. Minimum representative domain.

mode displacement parameters and $\eta$ the exponent of the Benzeggagh and Kenane criterion.

$\delta_{m}=\sqrt{\delta_{I}^{2}+\delta_{I I}^{2}}$

$\delta_{m 0}=\delta_{I 0} * \delta_{I I 0} \sqrt{\frac{\delta_{I}^{2}+\delta_{I I}^{2}}{\delta_{I}^{2} \delta_{I I 0}^{2}+\delta_{I I}^{2} \delta_{I 0}^{2}}}$

$\delta_{m f}=\frac{2}{K_{I I}{ }^{*} \delta m_{0}}\left[G_{I}+\left(G_{I I}-G_{I}\right)\left(\frac{\delta_{I I}^{2}}{\delta_{I}^{2}+\delta_{I I}^{2}}\right)^{\eta}\right]$

Combined damage $=\left(\frac{\delta_{m}-\delta_{m 0}}{\delta_{m}}\right)\left(\frac{\delta_{m f}}{\delta_{m f}-\delta_{m 0}}\right)$

In the same fashion as other authors [18,19], we consider mode II as predominant. We use the same stiffness value for the in plane and out of plane stiffnesses. The validity of this assumption will be demon strated in section 3 .

FEM discretization of the model was performed with Comsol ${ }^{\oplus}$ A Matlab script was used to launch Comsol with different constitutive parameters and store the results. The discretization was performed in the minimum representative domain (MRD) depicted in Fig. 2. Sym metry considerations were not applied due to the antisymmetry asso ciated with the shear.

In addition to the cohesive interfacial elements, 2D plane stress shell elements were used to model the matrix. A mapped rectangular mesh was used for better parametrization of the problem. The element sizes used for discretizing the nanofiller were one third of the nanofiller thickness in the transverse direction to accurately determine the na nofiller stress and strain distributions, and four times larger in the longitudinal direction to maintain a valid element aspect ratio. For the matrix, the size in the longitudinal direction was the same as that in the nanofiller and four times larger in the transverse direction to minimize the total number of elements while still allowing for sufficient accuracy.

The model was validated by comparing it with the experimental results obtained by Guo et al. [19]. In the experiment, the strain state was measured on a graphene sheet located at the free surface of an epoxy matrix, which was uniaxially strained at different strain levels. Fig. 3(a) shows that the correlation between the model and the ex perimental results is quite high. As there is only tensile stress in the longitudinal direction, there is symmetry in the damage and tensile stress distributions, while the shear stress is antisymmetric. Fig. 3(b) and (c) show the shear stress and damage distributions, respectively, which clearly explain the stress transfer mechanism occurring at the interface: shear stress arises from the matrix filler interface separation caused by the applied longitudinal strain. The amount of separation is governed by the longitudinal interface stiffness. Shear gradually loads the nanofiller causing a longitudinal stress to appear that grows to wards the nanofiller midpoint. If the value of shear stress reaches the shear strength, interfacial damage takes place. While damage increases for growing matrix nanofiller separation, shear stress will decrease as the tangential interface stiffness will be smaller due to the interfacial damage.

\section{Results and discussion. Influence of angular distortions and transverse strains on the interfacial stress transfer mechanisms}

We will qualitatively and quantitatively analyze how angular dis tortion and transverse strain affect the interfacial stress transfer me chanisms in a graphene epoxy nanocomposite. We first define a base case to describe its effect on the interfacial stress and damage dis tributions (section 3.1). Afterwards, we modify the constitutive para meters of the base case to analyze their sensitivity to angular distortion and transverse strain (section 3.2). The values for the base case have been selected from other well known works (for instance [19]) and correspond to those measured in actual graphene epoxy nanocompo sites:

- Matrix Young's Modulus $=3 \mathrm{GPa}$

- Graphene's Young's Modulus = $1 \mathrm{TPa}$

- Fracture energy: $G c=0.08 \mathrm{~N} / \mathrm{m}$;

- Tangential stiffness: $K_{I I}=74 \mathrm{TPa} / \mathrm{m}$;

- Graphene length: $l_{g}=10 \mu \mathrm{m}$;

- Graphene thickness: $h_{g}=10 \mu \mathrm{m}$;

- Weight fraction: $M_{f}=0.6 \%$;

- Tangential strength: $\tau=0.5 \mathrm{MPa}$;

In the case of a mode I fracture, which has been shown not to be a predominant mechanism $[18,19]$, we have used the following para meters, extracted from the mentioned works:

- Normal stiffness: $K_{I}=74000 \mathrm{TPa} / \mathrm{m}$;

- Normal strength: $\sigma=0.5 \mathrm{MPa}$;

For each parameter set, different combinations of longitudinal and transverse strains and/or angular distortions $\varepsilon_{\mathrm{X}}, \varepsilon_{\mathrm{Y}}$ and $\gamma_{\mathrm{xy}}$, respectively, were applied to the MRD free edges not corresponding to the filler (Fig. 2). The shear, longitudinal, transverse strains and damage dis tributions were then calculated. The equivalent displacement field to apply to each free matrix edge is defined by Equation (11) where $x$ and $y$ are the coordinates of each edge position where a displacement is applied.

$\left(\begin{array}{c}\Delta \mathrm{x} \\ \Delta y\end{array}\right)=\left[\begin{array}{cc}\varepsilon_{X} & \frac{\gamma_{x y}}{2} \\ \frac{\gamma_{x y}}{2} & \varepsilon_{Y}\end{array}\right]\left(\begin{array}{l}x \\ y\end{array}\right)$

3.1. Qualitative effect of angular distortion and transverse strain on the interfacial stress state

\subsubsection{Effect of angular distortion $\left(\gamma_{x y}\right)$ on the interfacial and nanofiller} stress states

Fig. 4 shows the effect of an applied angular distortion on the in terfacial damage and shear and nanofiller axial stress distributions in the base case. A significant influence is observed: when an angular distortion is applied, the interfacial shear stress distribution (Fig. 4(a)) shifts towards one of the interface ends, increasing its shear values. This implies that damage will take place (Fig. 4(b)) if the shear increase produced by the applied distortion reaches the shear strength value at some point in the interface. If damage already existed at some location, the applied distortion will increase its magnitude. In this case, shear at that location will not increase, as the maximum value, corresponding to the shear strength, has already been reached. The shear stress value will be governed by the cohesive model established. The damage increase 
a)
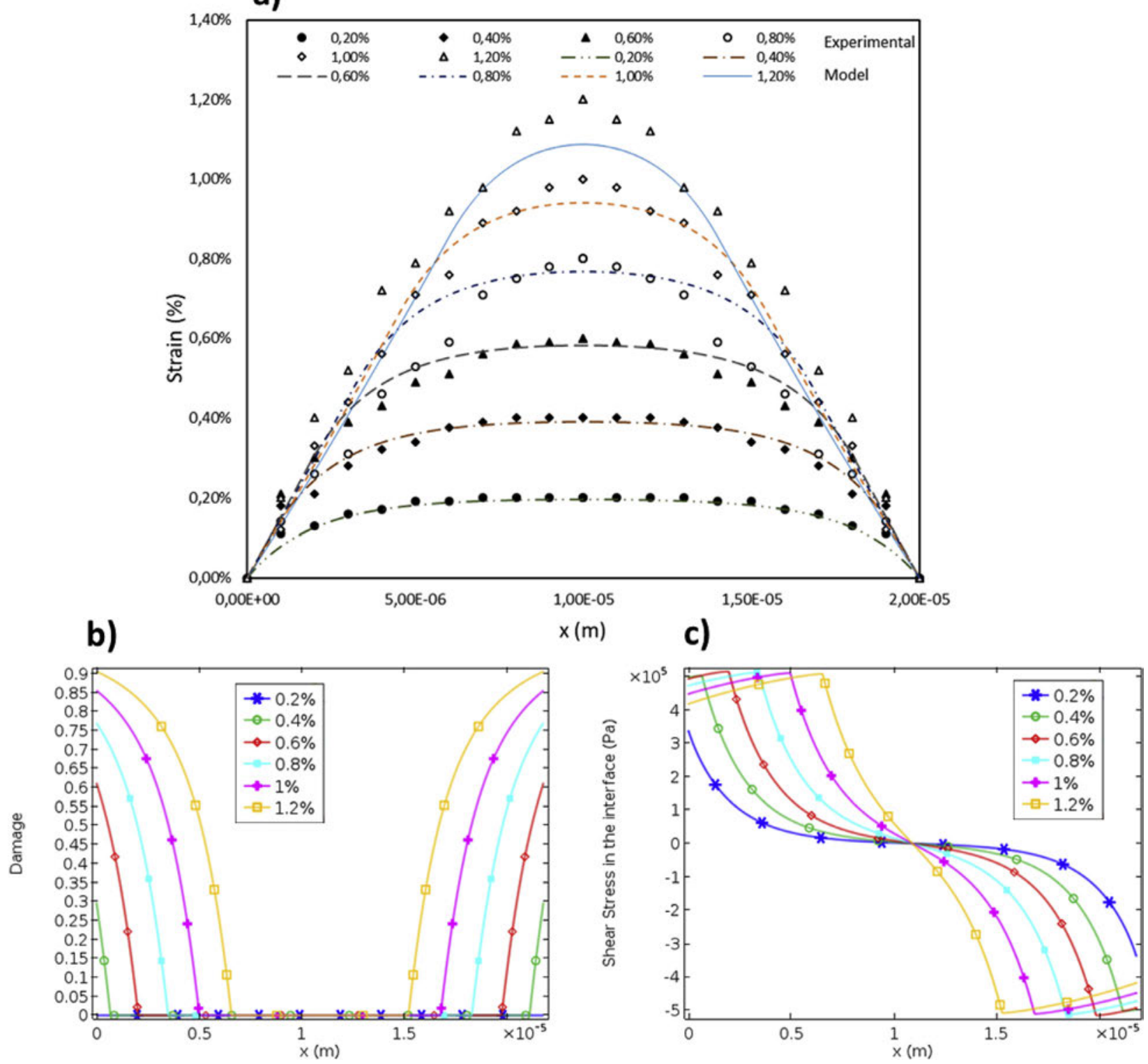

Fig. 3. (a) Guo et al. [19] experimental results vs. model. (b) Model damage and (c) model shear stress distributions.
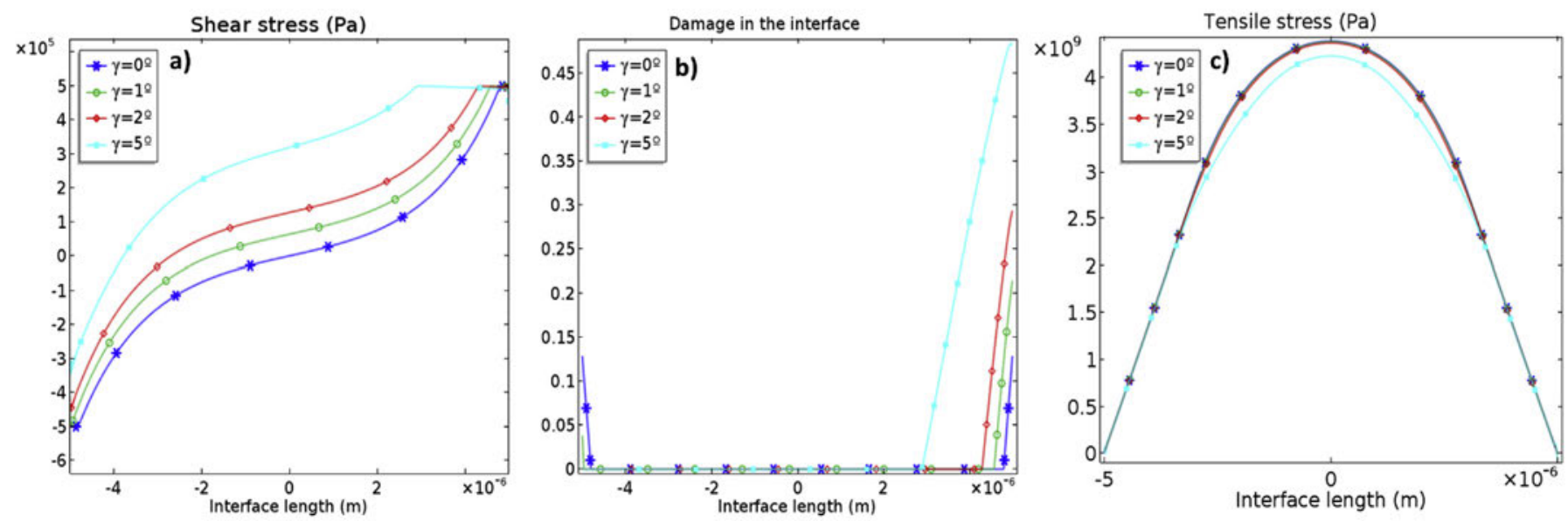

Fig. 4. Base case. Effect of an applied angular distortion on interfacial damage (a), interfacial shear stress (b) and nanofiller axial stress (c). 

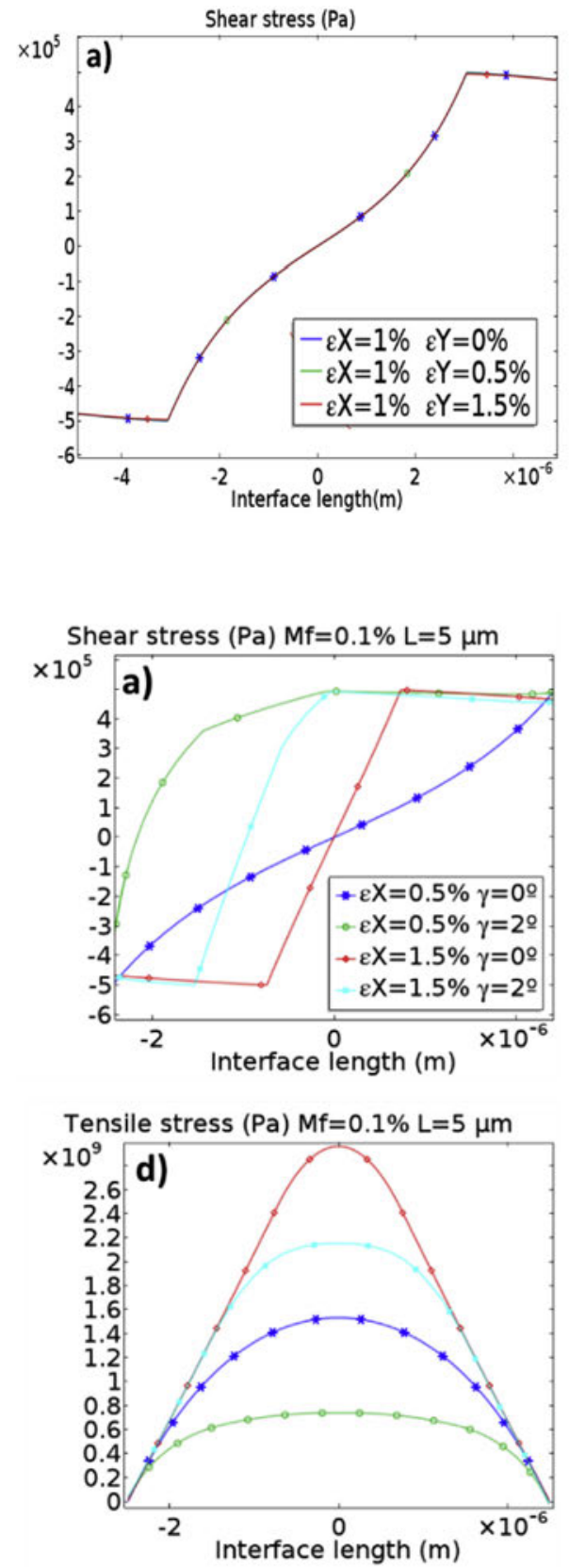
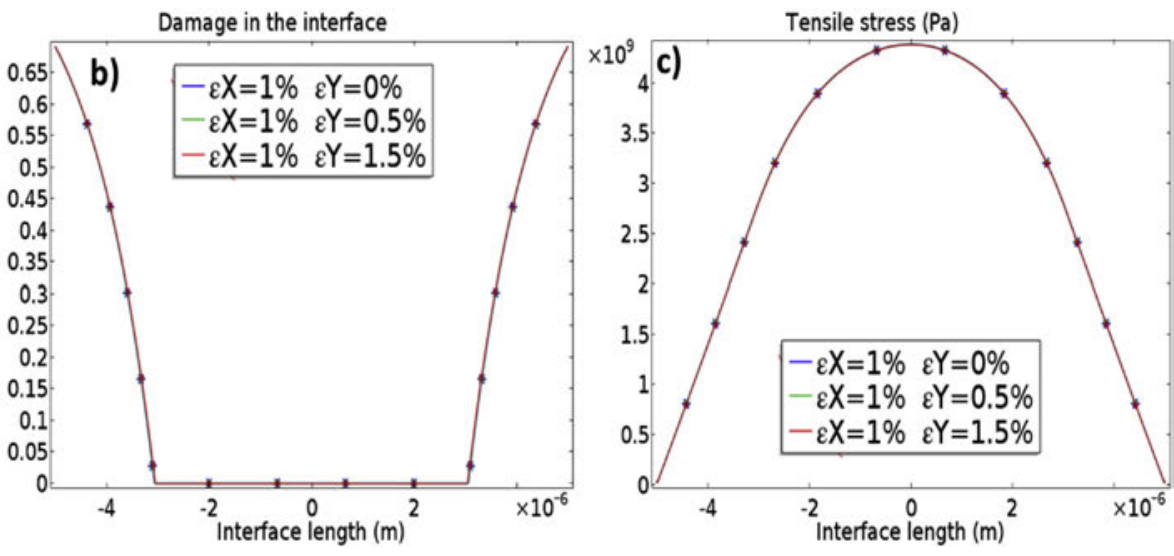

Fig. 5. Effect of transversal strain.
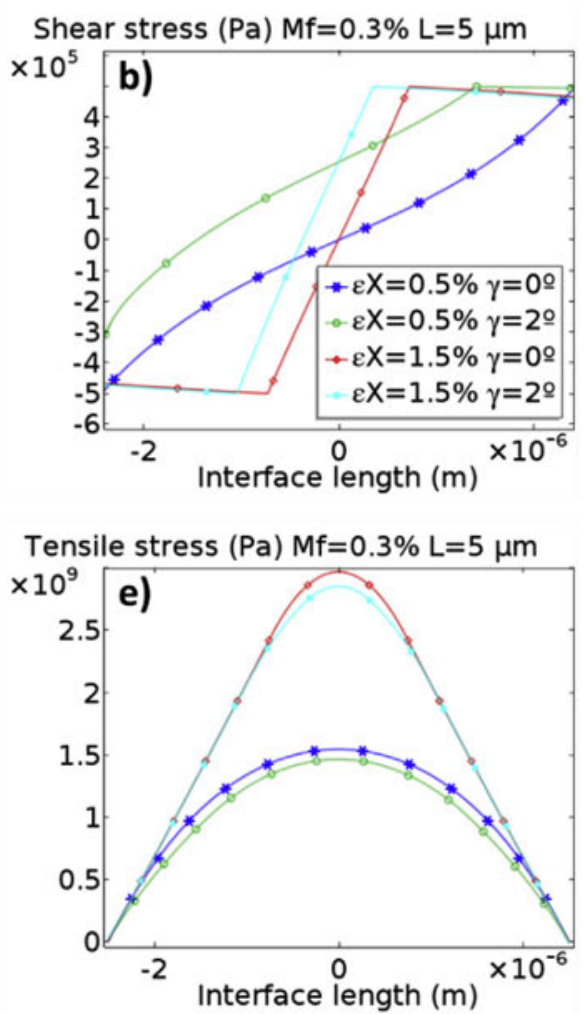

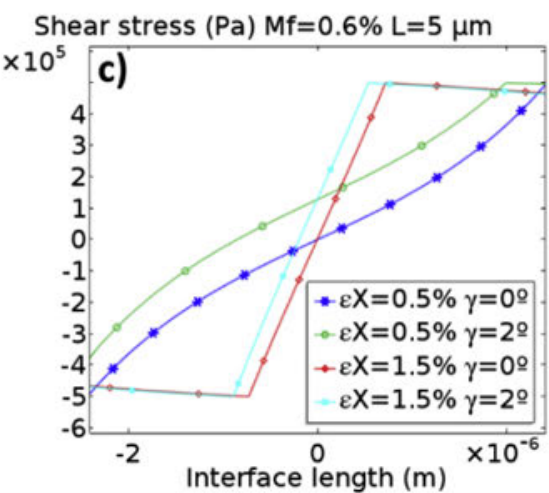

Tensile stress $(\mathrm{Pa}) \mathrm{Mf}=0.6 \% \mathrm{~L}=5 \mu \mathrm{m}$

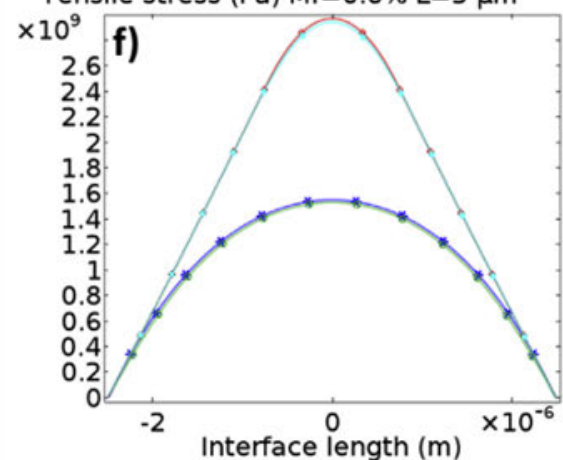

Fig. 6. Shear stress and tensile stress in the interface for filler length of $5 \mu \mathrm{m}$.

leads to a deterioration of the axial stress distribution (Fig. 4(c)) and consequently a reduction in its mean value. Since the homogenized, macroscale axial stiffness depends on the mean axial stress value, the nanocomposite stiffness will decrease as a result.

3.1.2. Effect of an applied transverse strain $\left(\varepsilon_{Y}\right)$ on the interfacial and nanofiller stress states

Fig. 5 shows the effect of three different levels of applied transverse strain on the interfacial shear stress distribution and the resulting in terfacial damage and nanofiller axial stress distributions in the base case. Even for a considerably large transverse strain of 1.5\%, the effect is not significant in comparison to that produced by applying an angular distortion or axial strain (Fig. 5(a), (b) and (c)). This is due to the large aspect ratio of the nanofiller. Since its thickness is much smaller than its length, the transverse interfacial separation produced by a given ap plied transverse strain is negligible in comparison to the longitudinal separation produced by an applied longitudinal strain of the same magnitude.

As pointed out in section 2, the above results are coherent with the assumptions suggested by other authors in the sense that mode II is the predominant mechanism for interfacial debonding [18,19]. In any event, the effect of angular distortions is clear and should not be ig nored.

3.2. Sensitivity of the effect of angular distortion and transverse strain to the interface constitutive parameters

As described in section 3.1, the shape of the stress and damage distributions is considerably modified by angular distortion. However, the level of modification greatly depends on the interfacial constitutive parameters. In this section we analyze how sensitive this effect is to the interface characteristics. 

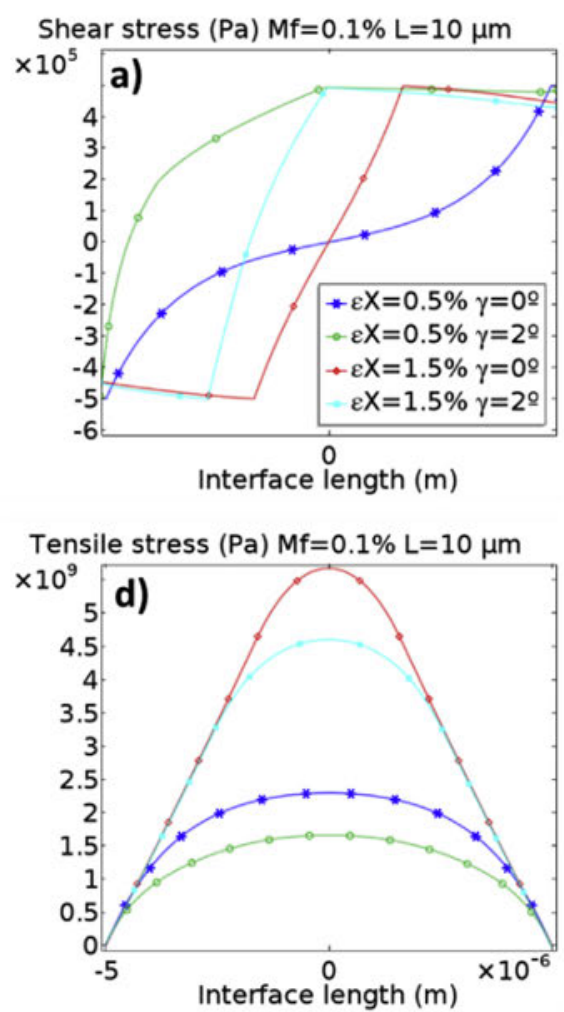

Shear stress $(\mathrm{Pa}) \mathrm{Mf}=0.3 \% \mathrm{~L}=10 \mu \mathrm{m}$

$\times 10^{5}$

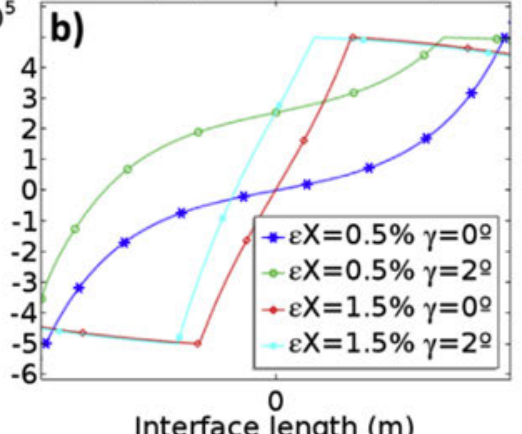

Tensile stress $(\mathrm{Pa}) \mathrm{Mf}=0.3 \% \mathrm{~L}=10 \mu \mathrm{m}$ $\times 10^{9}$

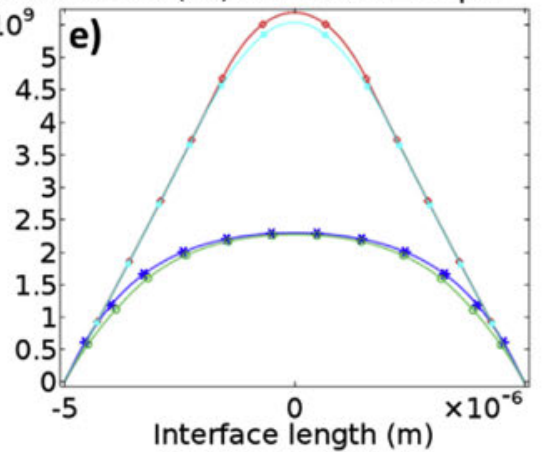

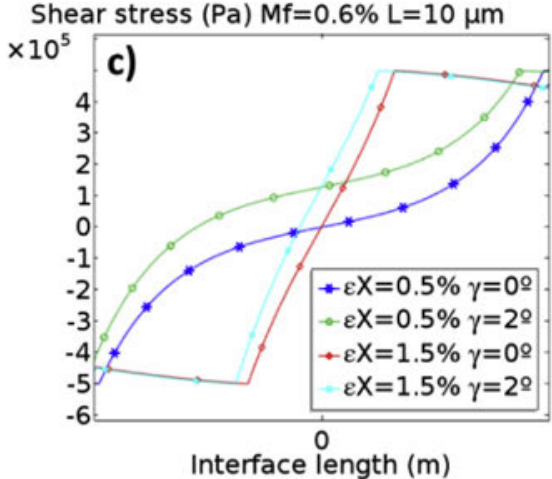

Tensile stress $(\mathrm{Pa}) \mathrm{Mf}=0.6 \% \mathrm{~L}=10 \mu \mathrm{m}$

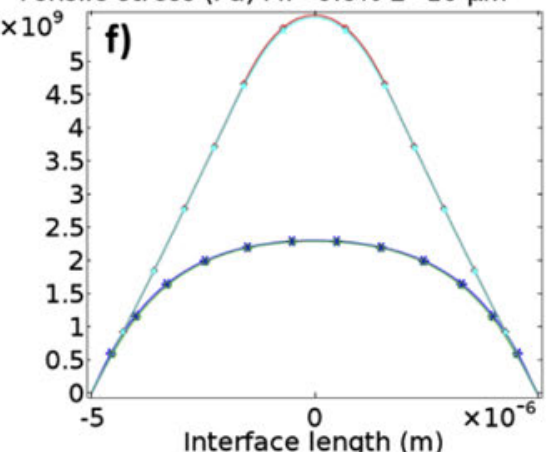

Fig. 7. Shear stress and tensile stress in the interface for filler length of $10 \mu \mathrm{m}$.
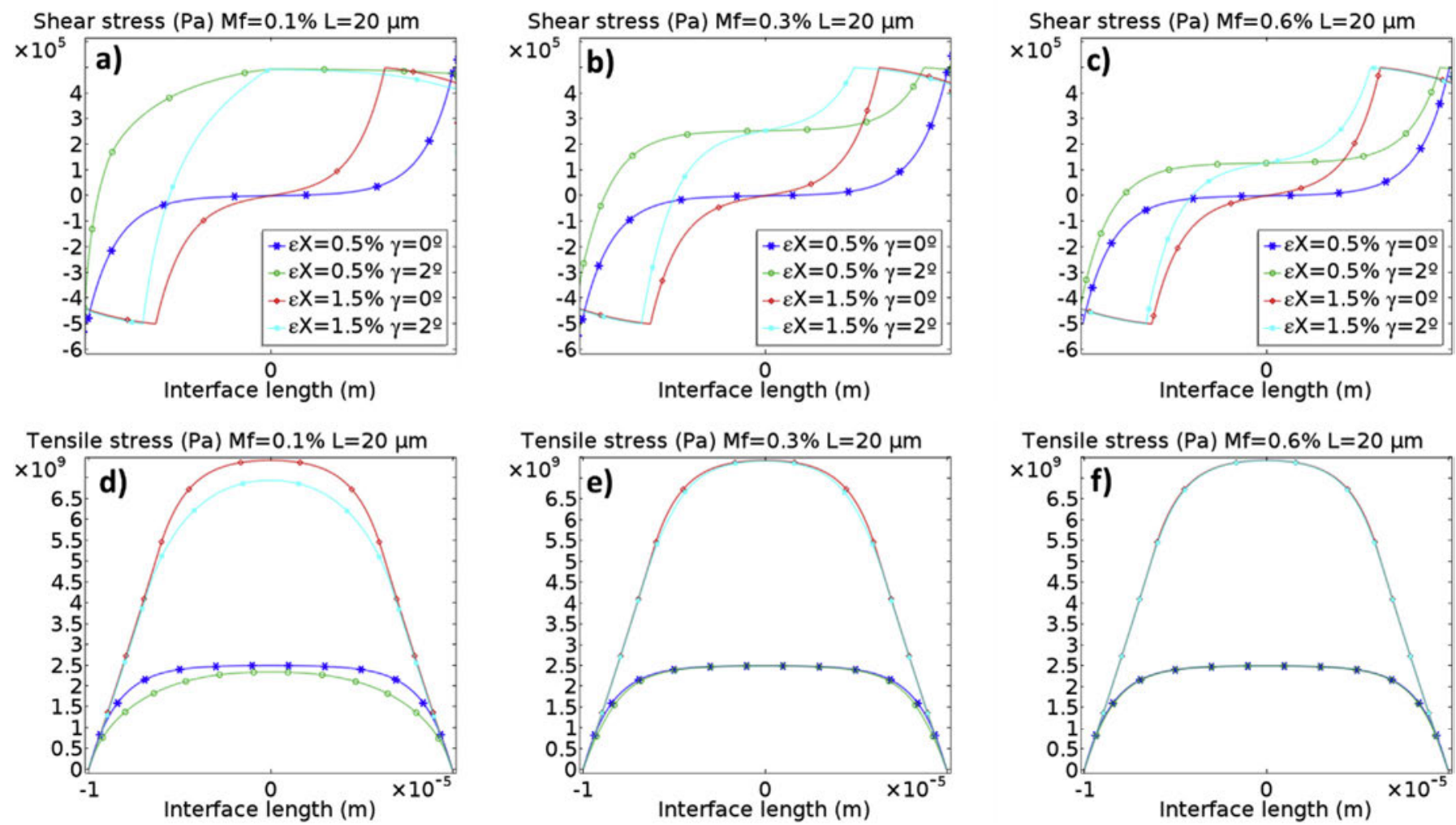

Fig. 8. Shear stress and tensile stress in the interface for filler length of $20 \mu \mathrm{m}$.

3.2.1. Filler content $\left(m_{f}\right)$ and filler length $\left(l_{f}\right)$

For this analysis, we have used lengths of $5 \mu \mathrm{m}$ (which is smaller than the critical length in a graphene epoxy interface) (Fig. 6), $10 \mu \mathrm{m}$ (critical length) (Fig. 7) and $20 \mu \mathrm{m}$ (greater than the critical length)
(Fig. 8). We have considered weight fractions of $0.1 \%, 0.3 \%$ and $0.6 \%$ for filler content, which are values commonly used in experimental analyses.

The effect of an applied angular distortion on the shear stress 

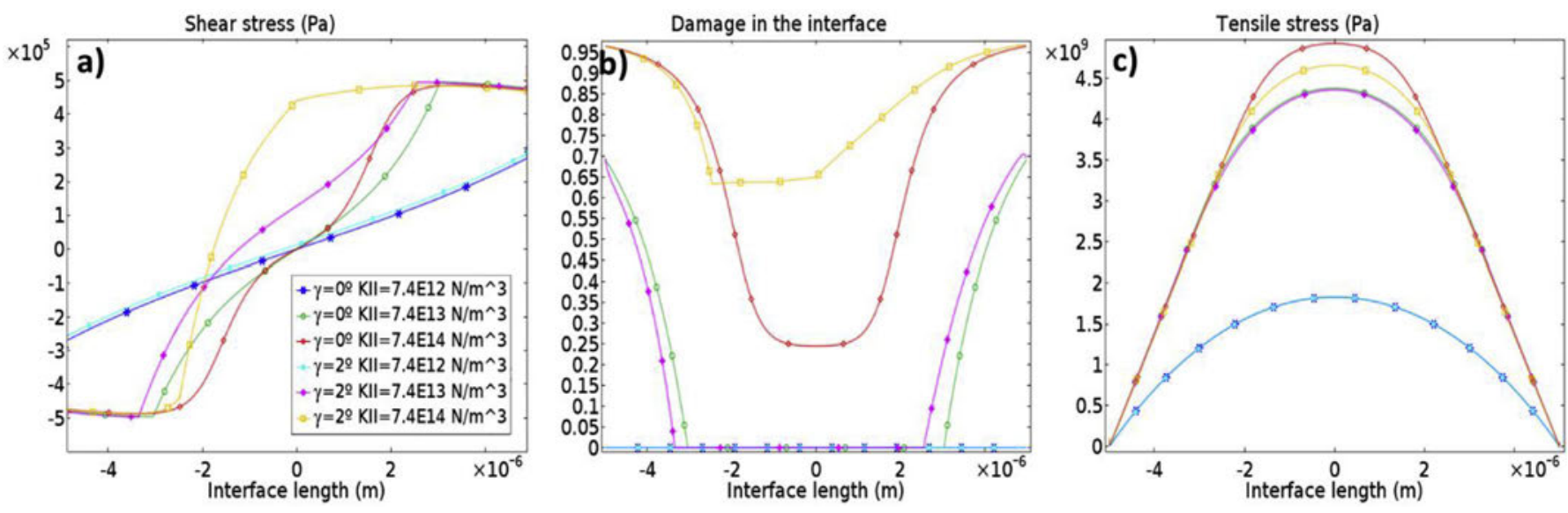

Fig. 9. Effect of the longitudinal stiffness.

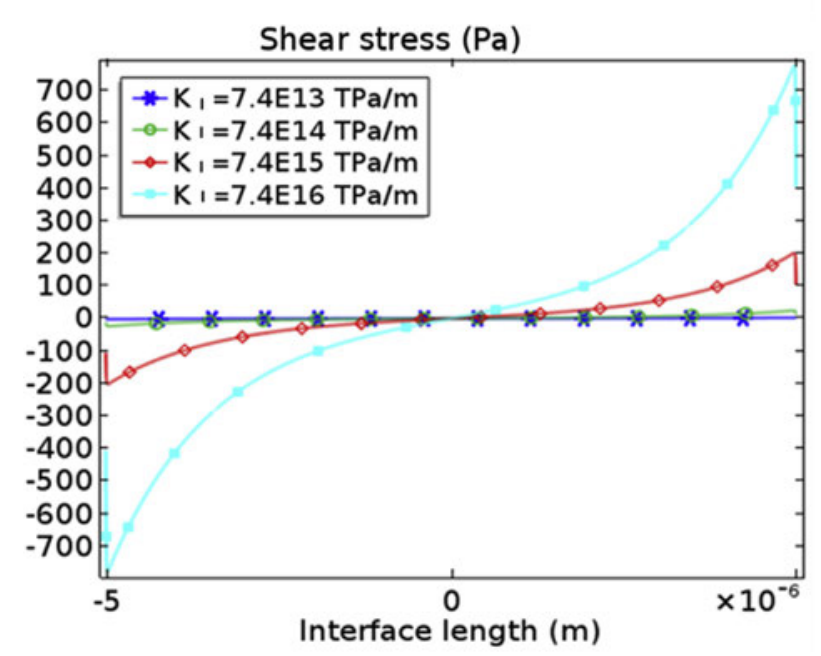

Fig. 10. Effect of transversal strain on the interface (2.5\% transverse strain).

distribution is more significant for small filler contents, and vanishes for larger ones, as subfigures (a) to (c) in Figs. 6, Figs. 7 and 8 show. This is due to the stiffening effect of the nanofiller. The resulting damage and axial stress distribution (and its mean value) are consequently affected, as shown in subfigures (d) to (f) in the same figures. The effect is also larger for small simultaneously applied axial strains since, if no dis tortion existed, the onset of damage would not have been reached at any point of the interface.
The effect of an applied angular distortion is much more notable for small lengths. This is the expected behavior, with a larger slope in the shear distribution due to a greater matrix filler longitudinal separation produced by a given applied strain, as the stiffening effect provided by the nanofiller is smaller.

\subsubsection{Interface longitudinal stiffness $\left(K_{I I}\right)$}

For a given strain state, a greater interface longitudinal stiffness will increase the values of the shear stress distribution, so the existing in terfacial damage will be increased as a result. Consequently, the effect of angular distortion will be magnified if the longitudinal stiffness in crease. This can be clearly observed in Fig. 9, where an applied long itudinal strain of $1 \%$ and a transverse strain of $1.5 \%$ are simultaneously applied. As described in section 3.1.2, the effect of the transverse strains is weak.

However, for large longitudinal stiffnesses, the effect can become evident, as it dramatically increases the damage at the filler midpoint (Fig. 9(b)). As damage is increased, the axial stress distribution will show lower values and consequently a low mean value, which implies a decrease in the homogenized axial stiffness.

\subsubsection{Interface transverse stiffness $\left(K_{I}\right)$}

The effect of $\mathrm{K}_{\mathrm{I}}$ is not quite as significant as that observed with the aforementioned parameters, since the substantial nanofiller length/ thickness ratio implies very small interfacial displacements even for large strains. The transverse separation's contribution to the damage energy is thus very small in comparison to that produced by the other strain states. Fig. 10 shows the effect of a large applied transversal strain of $2.5 \%$ for different interfacial transverse stiffnesses. Even for
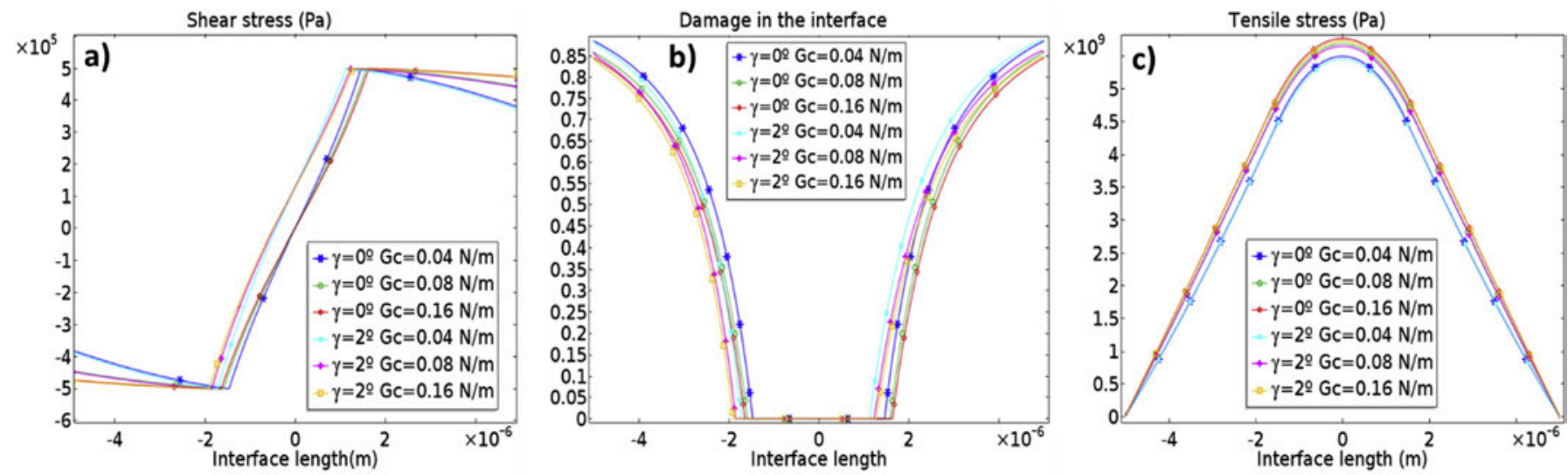

Fig. 11. Effect of fracture toughness on the interface. $\varepsilon_{\mathrm{x}}=1.5 \%$. 

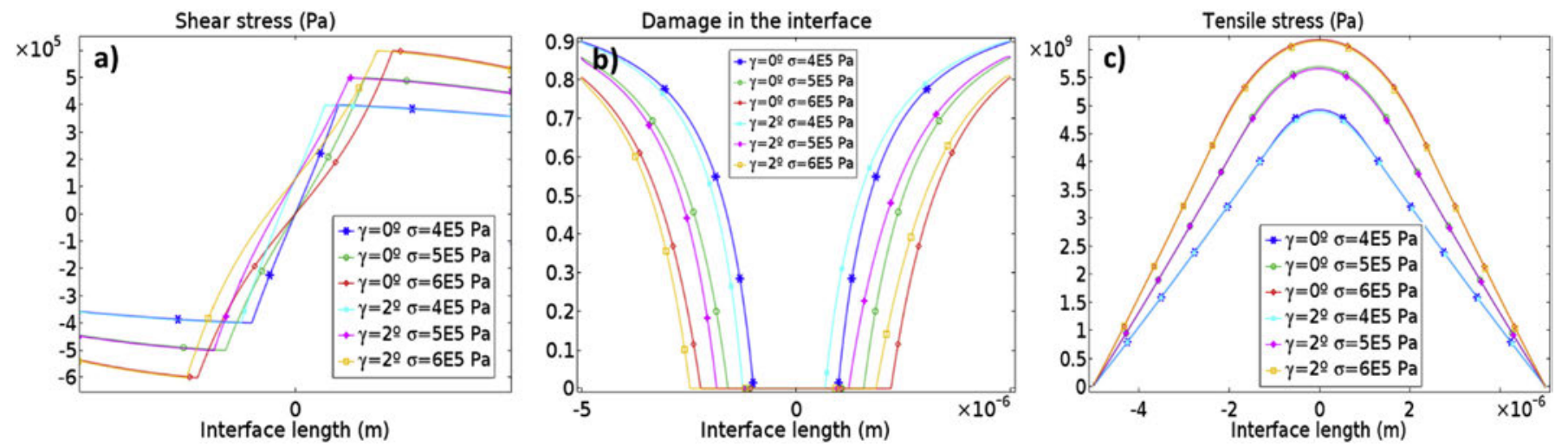

Fig. 12. Effect of shear strength on the interface. $\varepsilon_{\mathrm{x}}=1.5 \%$.
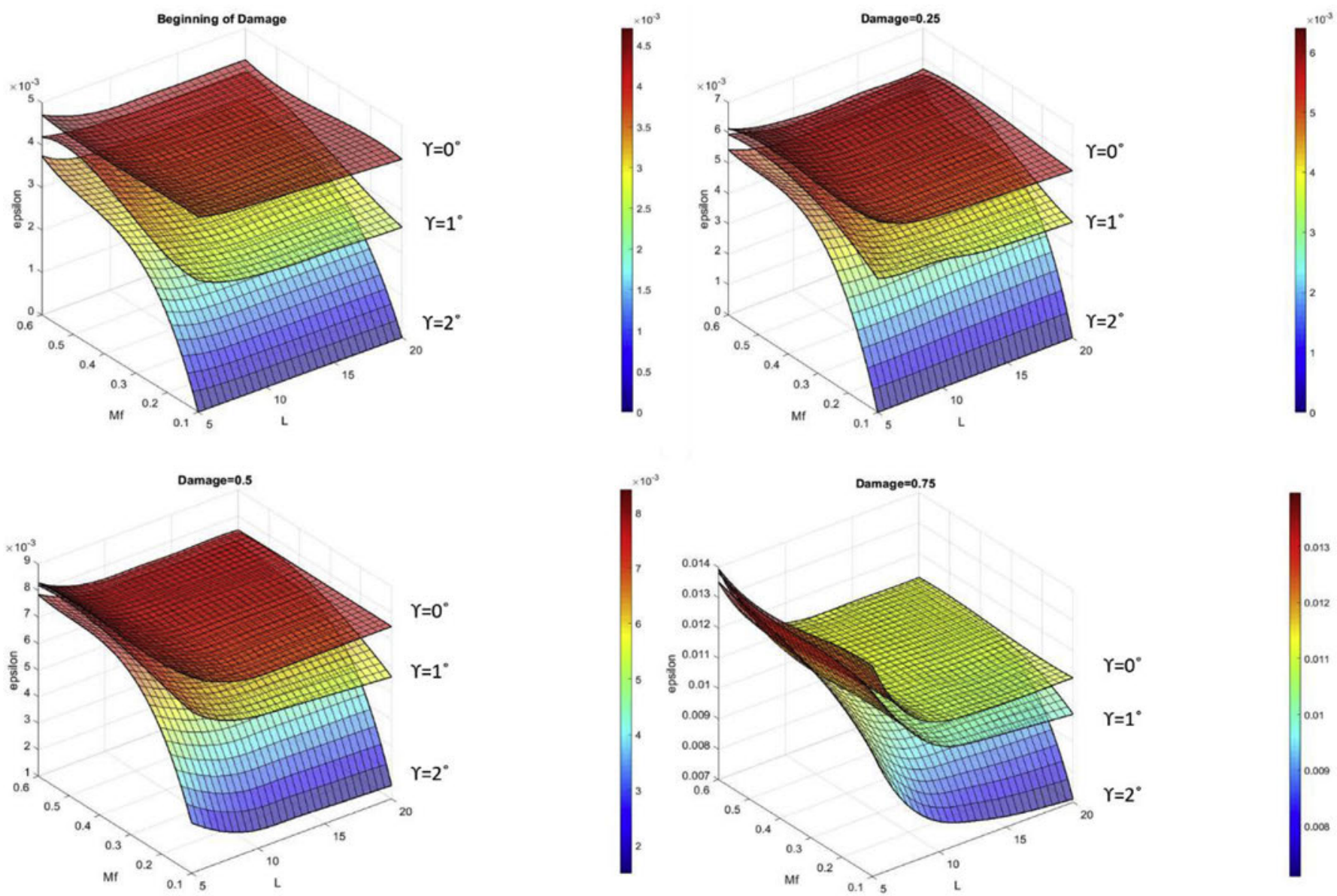

Fig. 13. Effect of angular distortion on the damage criterion.

the largest values, the shear stress caused by the applied transverse strain has a maximum value of $700 \mathrm{~Pa}$, far below the $0.5 \mathrm{MPa}$ needed for the initiation of debonding. Therefore, as pointed out in section 2, the effect of the transverse strain is not considered predominant, as the stress produced is small with respect with those produced by other strains. The damage figure is not shown, as the damage is inexistent and the tensile stress distribution is the same in all cases.

\subsubsection{Fracture toughness $(G)$}

Fig. 11 shows that the effect of an applied angular distortion is not sensitive to variations in the fracture energy. If damage is present, fracture energy will govern the value of the shear stress appearing at the damaged zone. As shown in Fig. 11(a), the influence of the fracture energy on the shear values at the damaged zones is small. Increasing fracture energy implies a lower slope in the shear stress distribution at these zones, tending to zero as fracture energy tends to infinity. How ever, the slope difference is small. Consequently, the changes in the tensile stress distribution and its mean value are small.

\subsubsection{Interface strength}

Greater shear strengths imply a larger maximum allowable shear at the interface and consequently a greater strain is necessary to produce the same damage. In theory, this would make the effect of an applied angular distortion clearer, as the appearance of additional shear would not imply increased damage. However, the effect is not notable even for shear strength increases of $50 \%$, as Fig. 12 shows. As explained in 

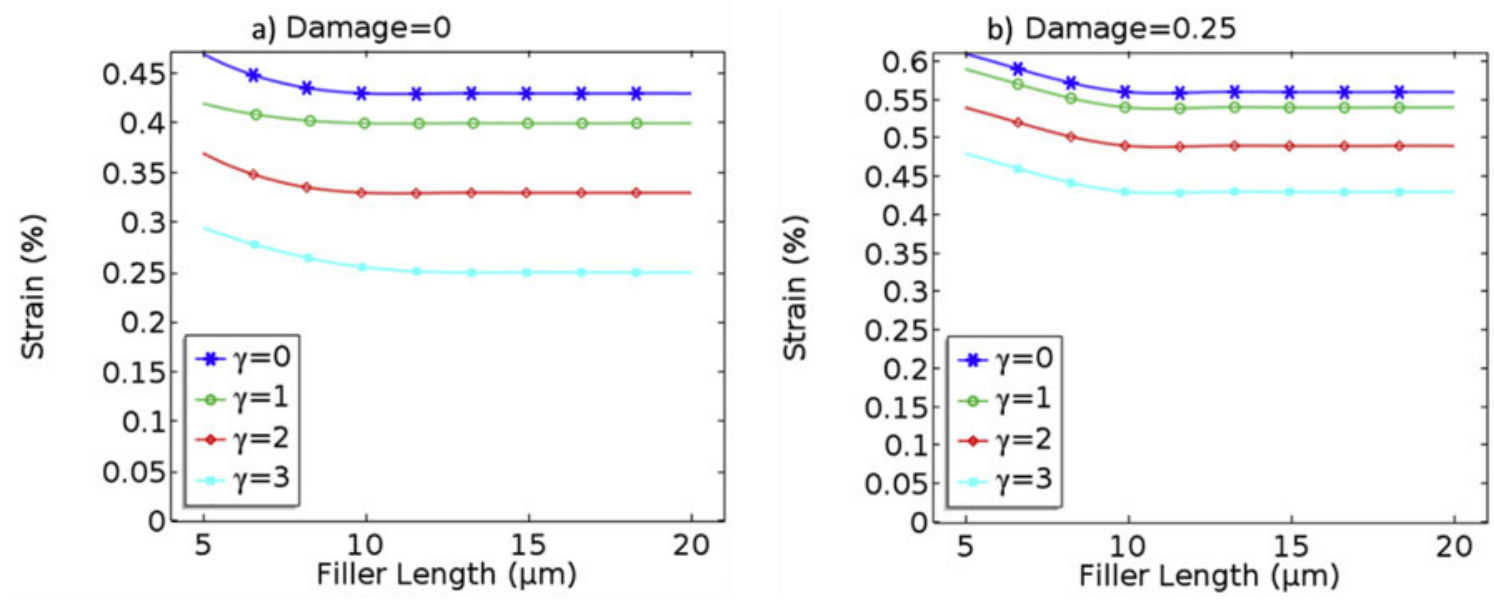

Fig. 14. Specific cuts from the damage maps.

section 3.1.2, the shift in the shear stress distribution produced by angular distortion leads to increased shear towards one end. An in crease in the shear strength leads to larger absolute shear values, but the increase in the slope of the tensile stress distribution is not sub stantial.

\section{Macroscale design maps including shear and transverse strains}

We have demonstrated that transverse strain and especially angular distortion have a significant influence on the interfacial stress transfer mechanisms at the nanoscale, by increasing the interfacial stresses and consequently reducing the onset of damage. This reduces the slope of the nanofiller tensile stress distribution and lowers its mean value as a result, leading to a smaller homogenized stiffness. If this fact is not considered when establishing failure criteria at the macroscale, the macroscale strain state limit could be incorrectly defined.

To assess the safety factor at the nanoscale with respect to the da mage produced for a given strain state including angular distortion, we present the maps shown in Fig. 13. This can be used as a tool to de termine the onset of damage when using a nanocomposite with given constitutive parameters (nanofiller length, filler contents, interfacial characteristics). We have used the parameters specified for the base case in section 3.1 and extended the analysis to include two additional lengths and filler weight fractions.

As observed in the figures, when compared to only considering longitudinal strain, including angular distortion dramatically modifies the damage criterion as the strain state limit is considerably reduced. This effect is especially observable for small nanofiller contents, as the reinforcement effect is obviously smaller. For growing damages, the effect of the strain state is less significant as most of the interfacial damage has already occurred [34].

Fig. 14 shows a specific result from the maps for a filler weight fraction of $0.6 \%$. Observe that for an angular distortion of $2^{\circ}$ the longitudinal allowable strain limit is approximately $25 \%$ smaller than if it is not considered. The difference rises to $40 \%$ for an angular distor tion of $3^{\circ}$. This shows that its effects must be considered when estab lishing failure criteria at the macroscale. Results where shear strain is zero can be compared with the results obtained by Guo et al. [19].

\section{Conclusions}

Transverse strain and especially angular distortion have a sig nificant influence on the stress state at the nanoscale, reducing the onset of damage and consequently worsening the elastic properties of the nanocomposite. They should, therefore, be considered when es tablishing macroscale failure criteria, as multiaxial strain states will appear if, for instance, filler misalignment or stress concentrators exist. Few works have been found that consider these parameters.

Whereas transverse strain has a weak effect because the thickness of the nanofiller is small in comparison to its length, the effect of angular distortion on the interfacial stress state is substantial. It produces a shift in the shear stress distribution and increases shear towards one of the interface ends, which increases damage and reduces the stress trans ferred to the nanofiller.

Whereas the effect of an applied angular distortion is more notable for small filler contents, small fiber lengths and stiff matrix filler in terfaces, it is less sensitive to changes in transverse stiffness, interfacial strength or fracture energy, since the change in the shear values and consequently in the damaged zones is not large and the modification of the slope of the nanofiller stress distribution is thus small. This implies a weak influence on the mean value of the stress distribution and con sequently of the nanocomposite stiffness.

\section{References}

[1] Lee K-Y, Aitomäki Y, Berglund LA, Oksman K, Bismarck A. On the use of nanocellulose as reinforcement in polymer matrix composites. Compos Sci Technol 2014;105:15-27.

[2] Saheb DN, Jog JP. Natural fiber polymer composites: a review. Adv Polym Technol 1999;18(4):351-63.

[3] Stankovich S, Dikin DA, Dommett GHB, Kohlhaas KM, Zimney EJ, Stach EA, Piner RD, Nguyen ST, Ruoff RS. Graphene-based composite materials. Nature 2006;442:282-6.

[4] Mohan VB, Lau K-t, Hui D, Bhattacharyya D. Graphene-based materials and their composites: a review on production, applications and product limitations. Compos B Eng June 2018;142(1):200-20.

[5] D. R. Bortz, E. G. Heras and I. Martin-Gullon, "Impressive fatigue life and fracture toughness improvements in graphene oxide/epoxy composites," Macromolecules, 01/10/2012, Vol. 45(1), pp.238-245.

[6] Wan Y-J, Tang L-C, Yan D, Zhao L, Li Y-B, Wua L-B, Jiang J-X, Lai G-Q. Improved dispersion and interface in the graphene/epoxy composites via a facile surfactantassisted process. Compos Sci Technol 2013;82:60-8.

[7] Wan Y-J, Tang L-C, Gong L-X, Yan D, Li Y-B, Wu L-B, Jiang J-X, Lai G-Q. Grafting of epoxy chains onto graphene oxide for epoxy composites with improved mechanical and thermal properties. Carbon 2014;69:467-80.

[8] Tang L, Wan Y, Yan D, Pei Y, Zhao L, Li Y. The effect of graphene dispersion on the mechanical properties of graphene/epoxy composites. Carbon 2013;60:16-27.

[9] Shiu S-C, Tsai Jia-Lin. Characterizing thermal and mechanical properties of graphene/epoxy nanocomposites. Compos B Eng January 2014;56:691-7.

[10] Lin F, Xiang Y, Shen H-S. Temperature dependent mechanical properties of graphene reinforced polymer nanocomposites - a molecular dynamics simulation. Compos B Eng February 2017;111(15):261-9.

[11] Awasthi AP, Lagoudas DC, Hammerand DC. Modeling of graphene-polymer interfacial mechanical behavior using molecular dynamics," Modelling Simul. Mater Sci Eng 2009;17:37.

[12] Sevostianov I, Yilmaz N, Kushch V, Levin V. Effective elastic properties of matrix composites with transversely-isotropic phases. Int J Solid Struct 2005;42:455-76.

[13] Aluko O, Gowtham S, Odegard G. Multiscale modeling and analysis of graphene nanoplatelet/carbon fiber/epoxy hybrid composite. Compos B Eng December 2017;131:82-90. 
[14] Li Y, Seidel GD. "Multiscale modeling of the effects of nanoscale load transfer on the effective elastic properties of unfunctionalized carbon nanotube-polyethylene nanocomposites," Modelling Simul. Mater Sci Eng 2014;22:28.

[15] Quaresimin M, Schulte K, Zappalorto M, Chandrasekaran S. Toughening mechan isms in polymer nanocomposites: from experiments to modelling. Compos Sci Technol 2016;123:187-204.

[16] Chandrasekaran S, Sato N, Tölle F, Mülhaupt R, Fiedler B, Schulte K. Fracture toughness and failure mechanism of graphene based epoxy composites. Compos Sci Technol 2014;97:90-9.

[17] Ramdoum S, Fekirini H, Bouafia F, Benbarek S, Serier B, Feo L. Carbone/epoxy interface debond growth using the Contour Integral/Cohesive zone method. Compos B Eng June 2018;142:102-7.

[18] Heidarhaei M, Shariati M, Eipakchi H. Effect of interfacial debonding on stress transfer in graphene reinforced polymer nanocomposites. Int J Damage Mech 2017;0(0):1-23.

[19] Guo G, Zhu Y. Cohesive-shear-lag modeling of interfacial stress transfer between a monolayer graphene and a polymer substrate. J. Appl. Mech. March 2015;82.

[20] Gong L, Kinloch IA, Young RJ, Riaz I, Jalil R, Novoselov KS. Interfacial stress transfer in a graphene monolayer nanocomposite. Adv Mater 2010;22(24):2694-7.

[21] Shen Ming-Yuan, Chang Tung-Yu, Hsieh Tsung-Han, et al. Mechanical properties and tensile fatigue of graphene nanoplatelets reinforced polymer nanocomposites. J Nano 2013;2013(4):565401. 9 https://doi.org/10.1155/2013/565401.

[22] Ang KK, Ahmed KS. An improved shear-lag model for carbon nanotube reinforced polymer composites. Composites 2013;50:7-14.

[23] Rahman R, Haque A. Molecular modeling of crosslinked graphene-epoxy nanocomposites for characterization of elastic constants and interfacial properties. Compos B Eng November 2013;54:353-64.
[24] Chen Z, Yan W. A shear-lag model with a cohesive fibre-matrix interface for analysis of fibre pull-out. Mech Mater 2015;91:119-35.

[25] Jiang T, Huang R, Zhu Y. Interfacial sliding and buckling of monolayer graphene on a stretchable substrate. Adv Funct Mat 2013;24(3):396-402.

[26] Lee J-U, Yoon D, Cheong H. Estimation of Young's modulus of graphene by Raman spectroscopy. Nano Lett 2012:4444-8.

[27] Cui S, Kinloch IA, Young RJ, Noe' L, Monthioux M. The effect of stress transfer within double-walled carbon nanotubes upon their ability to reinforce composites. Adv Mater 2009;21:3591-5.

[28] Xu Y, Li X, Wang X, Liang L. Inverse parameter identification of cohesive zone model for simulating mixed-mode crack propagation. Int J Solid Struct 2014;51:2400-10.

[29] Chen X, Deng X, Sutton MA, Zavattieri P. An inverse analysis of cohesive zone model parameter values for ductile crack growth simulations. Int J Mech Sci 2014;79:206-15.

[30] Belabed Z, Houari MSA, Tounsi A, Mahmoud S, Bég OA. An efficient and simple higher order shear and normal deformation theory for functionally graded materia (FGM) plates. Composites 2014;60:274-83.

[31] Lee MJ, Cho TM, Kim WS, Lee BC, Lee JJ. Determination of cohesive parameters for a mixed-mode cohesive zone model. Int J Adhesion Adhes 2010;30:322-8.

[32] Reeder JR, C Jr. JH. Mixed-mode bending method for delamination testing. AIAA J 1990;28(7):1270-6.

[33] Camanho P, Davila C, Moura MD. Numerical simulation of mixed-mode progressive delamination in composite materials. J Compos Mater 2003;16(37):1415-38.

[34] Ryu S-K, Jiang T, Im J, Ho PS, Huang R. Thermomechanical failure analysis of through-silicon via interface using a shear-lag model with cohesive zone. IEEE Trans Device Mater Reliab 2014;14(1):318-26. 